Case Report

\title{
Tuberculosis-Associated Immune Reconstruction Inflammatory Syndrome (TB-IRIS) in HIV-Infected Patients: Report of Two Cases and the Literature Overview
}

\author{
Klaudija Viskovic ${ }^{1}$ and Josip Begovac ${ }^{2}$ \\ ${ }^{1}$ Department of Radiology and Ultrasound, University Hospital for Infectious Diseases, Mirogojska Cesta 8, 10000 Zagreb, Croatia \\ ${ }^{2}$ Center for HIV/AIDS Infection, University Hospital for Infectious Diseases and School of Medicine, University of Zagreb, Croatia \\ Correspondence should be addressed to Klaudija Viskovic; viskovick@gmail.com
}

Received 23 February 2013; Accepted 31 March 2013

Academic Editors: A. Marangoni, S. Talhari, and X. Vallès

Copyright ( $) 2013 \mathrm{~K}$. Viskovic and J. Begovac. This is an open access article distributed under the Creative Commons Attribution License, which permits unrestricted use, distribution, and reproduction in any medium, provided the original work is properly cited.

\begin{abstract}
We describe two HIV-infected patients with tuberculosis-associated immune reconstruction inflammatory syndrome (TB-IRIS): one with "paradoxical" IRIS and the other with "unmasking" IRIS. TB-IRIS in HIV-infected subjects is an exacerbation of the symptoms, signs, or radiological manifestations of a pathogenic antigen, related to recovery of the immune system after immunosuppression. We focused on the radiological characteristics of TB-IRIS and the briefly literature review on this syndrome.
\end{abstract}

\section{Introduction}

Tuberculosis- (TB-) associated immune reconstruction inflammatory syndrome (TB-IRIS) is emerging as an important early complication of combination antiretroviral therapy (CART) in patients with HIV infection all over the world. In Europe, and United States studies the reported incidence of TB-IRIS was from $11 \%$ to $45 \%$ [1-4]. The frequency of TB-IRIS among Croatian patients in the study of Puljiz and colleagues in 2006 was $40.7 \%$ [5].

IRIS is a condition that results from rapid restoration of pathogen-specific immune responses to opportunistic infections, causing either the deterioration of a treated infection or the new presentation of a previously subclinical infection [6, 7]. There is no diagnostic test for IRIS, and a confirmation of the disease relies heavily upon case definitions incorporating clinical and laboratory data [7]. Case definitions for IRIS were published by French and collaborators in 2004 and by Shelburne and colleagues in 2006 [8,9]. A case definition specific for TB-IRIS was also suggested by Colebunders et al. in 2006 [10]. However, clinical management and research on IRIS were hindered by the lack of consensus case definitions and definitions that are specific to particular opportunistic infections [7]. To address this issue, in resource-limited settings, an international meeting of researches working in this field was convened in Kampala, Uganda in November 2006, and the international network for the study of HIVassociated IRIS (INSHI) was formed [7]. INSHI published criteria for "paradoxical" TB-IRIS and "unmasking" TBIRIS diagnosis in 2008 aimed for use in settings in which laboratory infrastructure is often limited [7].

The phenomenon of "paradoxical" reaction during the treatment of TB in which existing disease may worsen or new lesions appear has been recognized for many years, before the association with HIV coinfection [11]. Most reported cases have complicated the treatment of lymph node or cerebral disease, with enlargement of nodes seen in approximately $30 \%$ in one large series $[12,13]$. In the past few years an increase in the frequency and severity of paradoxical reactions in association with HIV coinfection has been reported both in patients receiving CART and those not on CART [1].

A diagnosis of "paradoxical" IRIS required both a diagnosis of TB and an initial response to antituberculous therapy (ATT) together with at least one of four major or two of three minor clinical criteria and exclusion of alternative explanations (Table 1) $[7,14]$.

The INSHI definition of CART-associated "unmasking" $\mathrm{TB}$ requires three criteria: not receiving $\mathrm{TB}$ treatment at 
TABLE 1: Case definition for "paradoxical" tuberculosis-associated immune reconstruction inflammatory syndrome (TB-IRIS) for use in resource-limited settings [7].

Three components to this case definition:

(1) Antecedent requirements (both of the following requirements must be met):

(1) Diagnosis of TB

Made before starting of combination antiretroviral therapy (CART) and should fulfill WHO criteria

(2) Initial response to TB treatment

The patient's condition should have stabilized or improved on appropriate TB treatment before CART initiation

(2) Clinical criteria

The onset of TB-associated IRIS manifestations should be within 3 months of CART initiation, reinitiation, or regimen change because of treatment failure

Of the following, at least one major criterion or two minor clinical criteria are required:

$$
\text { Major criteria }
$$

New or enlarging lymph nodes, cold abscesses, or other tissue involvement

New or worsening radiological features of TB

New or worsening central nervous system CNS TB

New or worsening serositis

\section{Minor criteria}

New or worsening constitutional symptoms such as fever, night sweats, or weight loss

New or worsening respiratory symptoms as cough, dyspnea, or stridor

New or worsening abdominal pain accompanied by peritonitis, hepatomegaly, splenomegaly, or abdominal adenopathy

(3) Excluded alternative explanations for clinical deterioration

Failure of TB treatment because of TB drug resistance

Poor adherence to TB treatment

Another opportunistic infection or neoplasm

Drug toxicity or reaction

TABLE 2: Provisional case definition for "unmasking" tuberculosis-associated immune reconstruction inflammatory syndrome (TB-IRIS) in resource-limited settings [7].

"Unmasking" TB-IRIS (provisional)

The following could suggest a diagnosis of "unmasking" TB-associated IRIS:

Patient is not receiving treatment for TB when CART is initiated and then presents with active TB within 3 months of starting CART

And one of the following criteria must be met:

(1) Heightened intensity of clinical manifestations, particularly if there is evidence of a marked inflammatory component to the presentation. Examples include TB lymphadenitis or TB abscesses with prominent acute inflammatory features, presentation with pulmonary TB that is complicated by respiratory failure due to ARDS, and those who present with a marked systemic inflammatory distress syndrome related to TB

(2) Once established on TB treatment, a clinical course that is complicated by a paradoxical reaction

CART initiation; diagnosis of active TB after CART initiation; fulfilling World Health Organization (WHO) diagnostic criteria for TB [7]. Additional criteria required for "unmasking" TB-IRIS are presentation within three months of CART initiation and either "heightened intensity of clinical manifestations or development of a paradoxical reaction once on TB treatment (Table 2) $[7,14,15]$.

In this case report and overview of the literature, we present the disease spectrum of "paradoxical" and "unmasking" TB-IRIS in two patients with complete resolution of disease.

\section{Patients and Methods}

Both patients received medical care at the University Hospital for Infectious Diseases (UHID) in Zagreb, Croatia, which provides comprehensive care of all HIV-infected patients in Croatia. Croatia has a low-level HIV epidemic, and there were 792 registered HIV/AIDS cases at the end of 2009, according to the HIV/AIDS Register of the Croatian National Institute of Public Health $[16,17]$. The definition of TB-IRIS in our patients required a case definition criterion for "paradoxical" TB-IRIS that consists of three components as displayed in 
Table 1 or fulfilling criteria of "unmasking" TB-IRIS displayed in Table 2 [7]. Informed consent was obtained from both patients granting us the permission to publish the details of their medical presentation and management.

\section{Case Presentations}

3.1. Case 1 Patient with "Paradoxical" TB-IRIS. A 53-yearold Caucasian female was admitted to UHID in December 2006 with a three-week history of fever and cough. HIV-1 infection was diagnosed in 2004, and CART was prescribed in April 2004. However, the patient stopped taking CART in December 2005.

At admission, chest radiographs showed patchy medial lobe infiltrate and massive right hilar adenopathy. Native and postcontrast thoracic computed tomography (CT) scan confirmed large right side mediastinal and pulmonary lymph nodes. High resolution CT scan of the right lung showed peripheral, poorly defined, small (2-4 mm diameter) centrilobular nodules and branching linear opacities of similar caliber originating from a single stalk (the "tree-in-bud" pattern) [18]. No cavities were detected. Sputum cultures grew Mycobacterium tuberculosis (M. tuberculosis) sensitive to all ATT drugs.

Standard ATT (isoniazid, rifampin, pyrazinamide, and ethambutol) was initiated in December 2006 and was given for 8 weeks. After that, isoniazid and rifampin were given for the period of 12 months. CART (stavudine, lamivudine, and efavirenz) was given 15 days after initiation of ATT. In February 2007, three months after initiation of ATT, two "cold" abscesses of thoracic wall were noticed and confirmed by ultrasound (US) and CT imaging. We performed initial aspiration biopsy to establish the diagnosis and to exclude malignancy [19]. Eventually, surgical incision and drainage was done. Pulmonary infiltrates at that time were completely resolved. In August 2007, the patient noted enlarged cervical lymph nodes. On physical examination performed at that time, we found enlarged lymph nodes in the left anterior cervical chain and left supraclavicular region (Figure 1). The involved areas were minimally tender with no warmth or erythema. The largest lymph node was approximately $3 \times$ $4 \mathrm{~cm}$, and the consistency of the lymph nodes varied from firm to fluctuant. CT and US scans of the neck, performed in August 2007, revealed large left side lymphadenopathy along the anterior cervical chain and in the supraclavicular region. Most of the lymph nodes were noted to be necrotic. A US-guided fine needle aspiration of two largest, necrotic cervical nodes was performed. About $70 \mathrm{~mL}$ of necrotic, purulent material was removed. Cytological examination showed granulomatous inflammation with caseous necrosis. Acid-fast bacilli (AFB) were seen, but cultures did not grow M. tuberculosis. IRIS was treated with steroids (methylprednisolone) for 10 months from January to November 2007.

The CD 4 cell count and viral load (HIV RNA copies/mL) over time are presented in Figure 2.

The patient's health condition slowly improved over the next 12 months, and in August 2008, the neck lymphadenopathy and thoracic wall abscesses had resolved with only scar tissue remaining at the site of previous surgical incision.

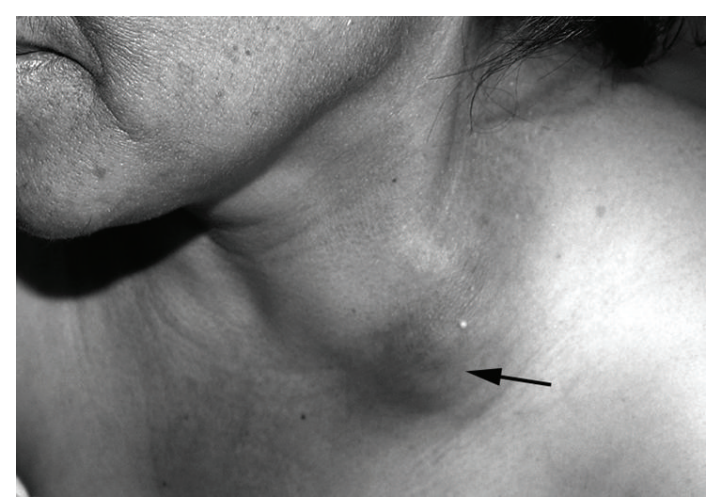

FIgURE 1: Enlarged cervical lymph nodes in a patient (Case no. 1) with "paradoxical" tuberculosis- (TB-) associated immune reconstruction inflammatory syndrome (TB-IRIS) (arrow).

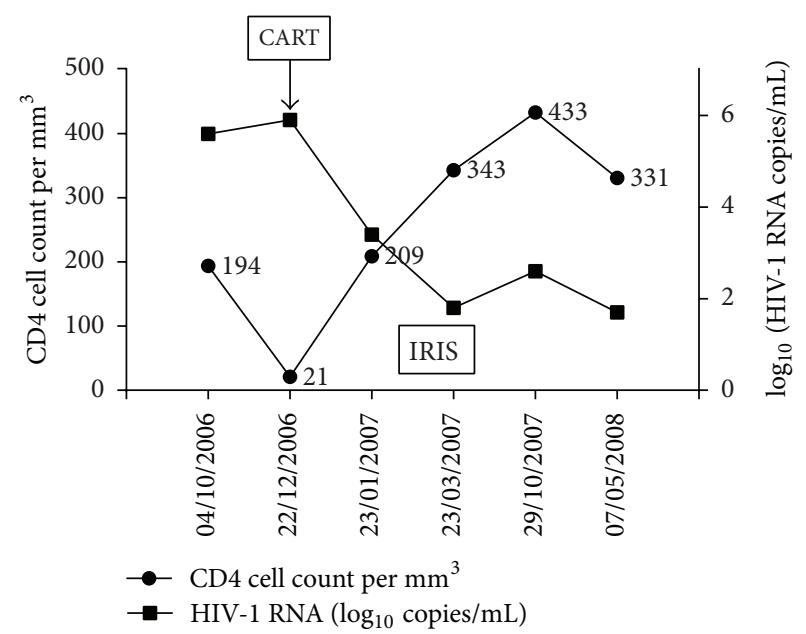

FIGURE 2: The CD4 cell count and viral load (HIV RNA copies/mL) over time in a patient with "paradoxical" tuberculosis- (TB-) associated immune reconstruction inflammatory syndrome (IRIS). CART: combination antiretroviral therapy.

3.2. Case 2 Patient with "Unmasking" TB-IRIS. A 40-year-old Caucasian male came to a routine followup at the HIV/AIDS Outpatient Center at the University Hospital for Infectious Diseases (UHID) in Zagreb in February 2008. On physical examination, a painless mass in the left inguinal region was noted. The skin overlying the region was not erythematous, and the patient had no fever. He stated having intermittent low-grade fever and a minor swelling in the left groin since October 2007.

He was known to be HIV infected after a tonsillectomy performed in May 2007. Pathohistological examination of the tonsils revealed chronic granulomatous infection. At that time, he had a reactive purified protein derivative (PPD) test which was positive (measured $18 \times 20 \mathrm{~mm}$ ). The chest $\mathrm{X}$-ray was normal. CART was commenced with abacavir, lamivudine, and efavirenz in July 2007. No TB prophylaxis was given.

In a car accident in August 2007, he had lumbar region contusion, and eventually the lumbar spine CT scan was 


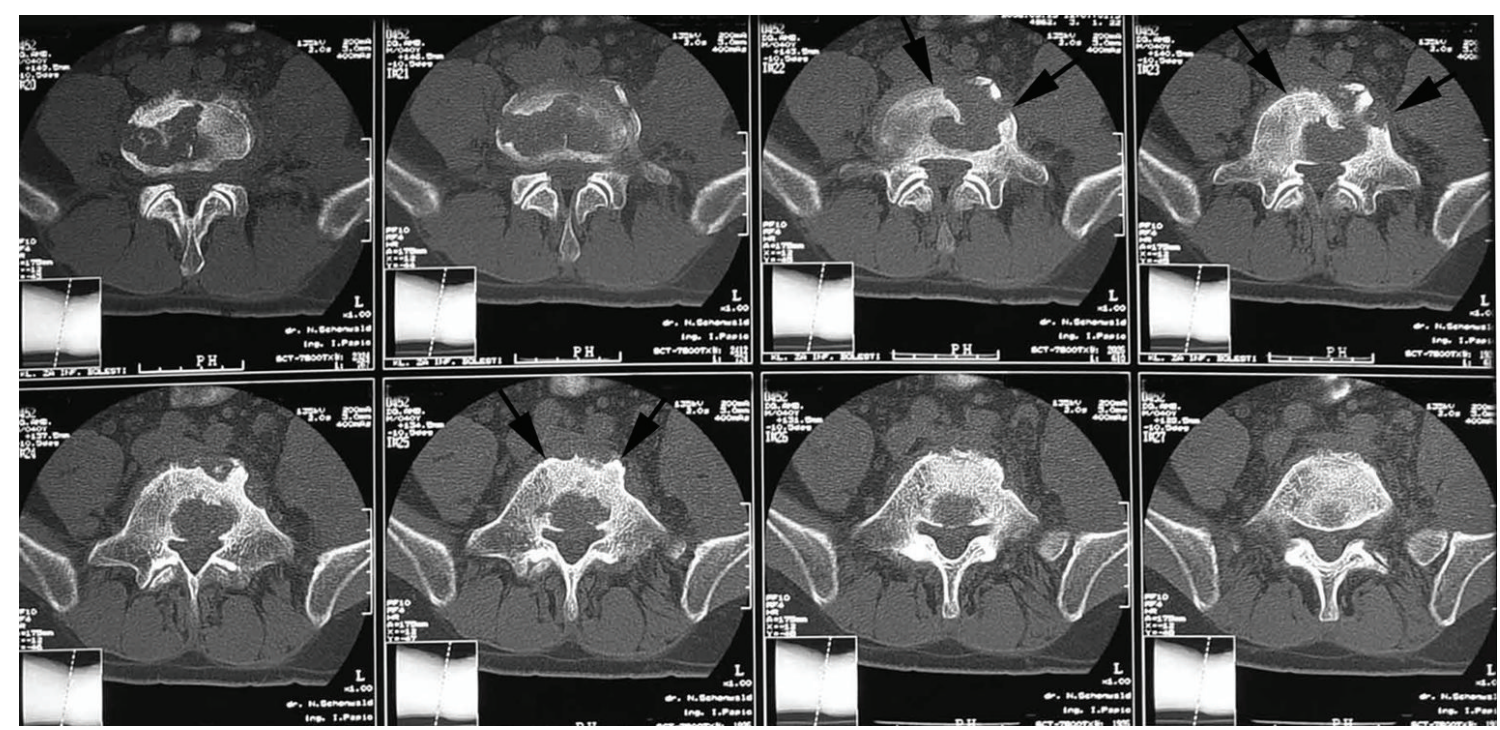

Figure 3: CT scans demonstrated permeative bone destruction of the lower end plate of the fourth lumbar vertebra and the upper end plate of the fifth lumbar vertebra, consistent with spondylodiscitis in a patient with "unmasking" tuberculosis- (TB-) associated immune reconstruction inflammatory syndrome (TB-IRIS) (arrows) (Case no. 2).

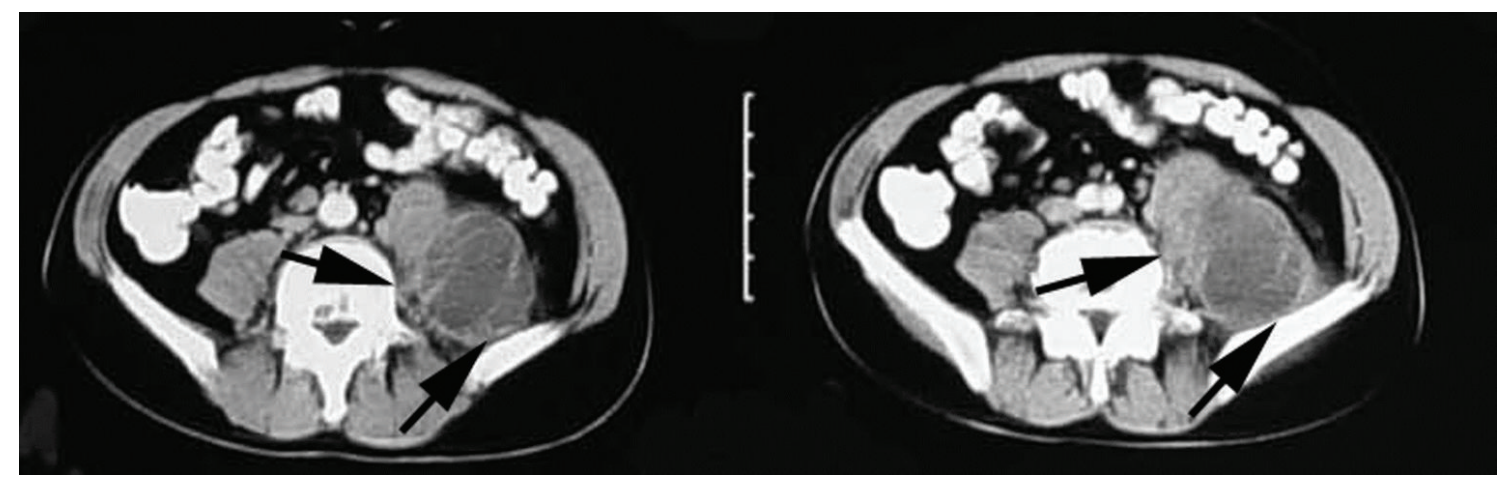

FIGURE 4: Axial, contrast-enhanced abdominal computed tomography (CT) scans showed huge abscess in a left psoas muscle (arrows) in a patient with "unmasking" tuberculosis- (TB-) associated immune reconstruction inflammatory syndrome (TB-IRIS) (Case no. 2).

performed to exclude bone fractures. The CT scan showed no evidence of traumatic or other bone lesions of lumbar spine vertebrae. The paravertebral spaces were normal.

After the above mentioned visit in February 2008, the patient was admitted to UHID. Abdominal US revealed hypoechoic mass in the left psoas muscle extending in the left inguinal region above the inguinal ligament. Axial, contrast-enhanced lumbar spine and abdominal CT scans demonstrated permeative bone destruction of the lower end plate of the fourth lumbar vertebra and the upper end plate of the fifth lumbar vertebra, consistent with spondylodiscitis and a huge left psoas abscess (about $35 \times 10 \times 8 \mathrm{~cm}$ ) (Figures 3 and 4$)$.

A lumbar puncture was performed and was unremarkable. The chest radiograph was normal. The blood CD 4 cell count was $185 / \mathrm{mm}^{3}$, and the HIV plasma viral load was $<50$ copies per $\mathrm{mL}$. We present the blood CD4 cell count and HIV1 RNA response to CART over time in Figure 5.
US-guided aspiration of psoas abscess was performed, which revealed purulent material. AFB staining was positive, and subsequently $M$. tuberculosis was cultured. It was sensitive to all major AT drugs. The patient was subsequently referred to the Department of Surgery at the University Traumatology Clinic, Zagreb, where surgical drainage via an extra peritoneal approach was performed. The abscess was completely drained, and $1400 \mathrm{~mL}$ of pus was evacuated.

Treatment with rifampin, ethambutol, isoniazid and pyrazinamide was initiated in February 2008 and was given for 8 weeks. After that isoniazid, and rifampin were given for the period of 12 months.

Over the next nine month,s the patient had several further outpatient follow-up visits. A follow-up magnetic resonance imaging (MRI) examination of the spine was performed in September 2008. It showed high signal intensity of L4 and L5 lumbar vertebral bodies in T1- and T2-weighted images (WIs) as signs of healing, with loss of height from 


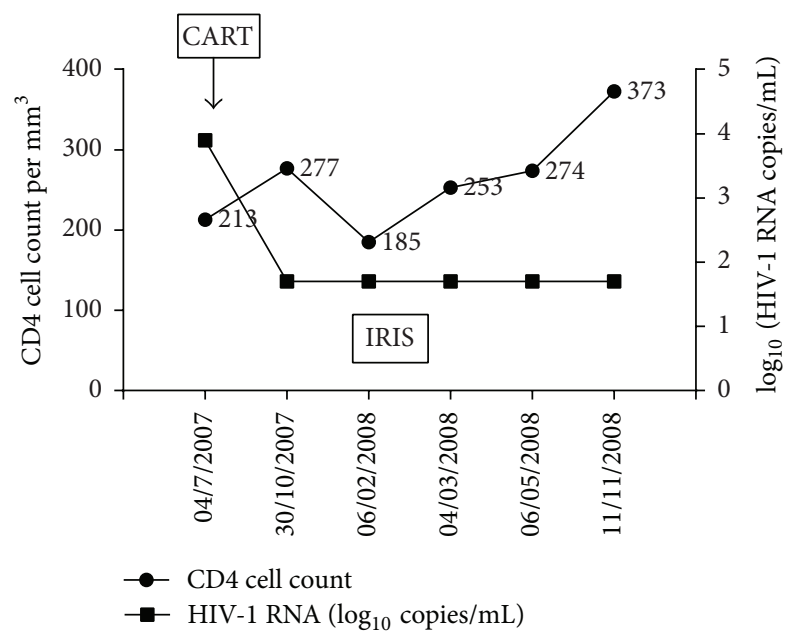

FIgURE 5: The blood CD4 cell count and HIV-1 RNA response to CART over time in a patient with "unmasking" tuberculosis- (TB-) associated immune reconstruction inflammatory syndrome (TBIRIS). CART: combination antiretroviral therapy.

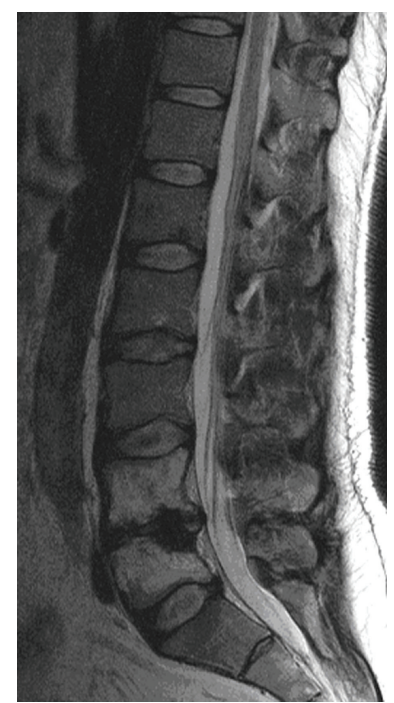

FIGURE 6: A follow-up magnetic resonance imaging (MRI) examination of the spine performed in September 2008 showed high signal intensity of L4 and L5 lumbar vertebral bodies in T2-weighted images (WIs) as signs of healing, with loss of height from infective destruction. There was no spinal canal stenosis.

infective destruction. There was no spinal canal stenosis (Figure 6). During the visit in November 2008, all signs and symptoms of TB resolved.

\section{Discussion}

The patient with "paradoxical" form of TB-IRIS fulfilled the INSHI criteria: the diagnosis of TB was made before reinitiation of CART and fulfilled WHO criteria for diagnosis of smear-positive pulmonary TB and extra pulmonary TB
[7]. The onset of TB-IRIS manifestation was within 3 months of CART initiation. The pulmonary infiltrate resolved after two months of ATT, but at the same time thoracic wall cold abscesses appeared, followed by enlarged, necrotic lymph nodes at the supraclavicular region and neck. We excluded possible alternative explanations for clinical deterioration: poor adherence to ATT because the patient was hospitalized and carefully monitored, and the presence of another opportunistic infection or neoplasm was excluded by different diagnostic methods. Drug resistance was excluded by testing with culture techniques [20].

The patient with spondylodiscitis and psoas abscess also fulfilled the INSHI [7] criteria for "unmasking" TB-IRIS. However, there is some uncertainty about the precise time point of the onset of TB symptoms in relation to CART initiation. Our patient recalled the first symptoms of TB infection about three months after CART initiation. However, CT scans of lumbar spine and abdomen were performed on his follow-up visit seven months after CART initiation and showed signs of spondylodiscitis and psoas abscess. Because of a car accident, a CT scan of lumbar vertebrae was performed one month after CART initiation. It was unremarkable; no signs of bone destruction or trauma were found. The destruction of lumbar spine vertebrae and psoas abscess gradually developed in the course of seven months. The marked inflammation that resembled a formation of large psoas abscess supports the diagnosis of IRIS.

At the time when the patient was found to be HIV infected, the PPD test was positive. Latent TB infection (LTBI) is defined by a positive M. tuberculosis-specific immune response in the PPD or an interferon-gamma release assays (IGRAs) in the absence of active TB. A positive PPD test indicates an immunological memory to previous or ongoing contact with $M$. tuberculosis and may be found also in patients who were BCG vaccinated or who had contact with atypical mycobacteria [21]. Our patient was BCG vaccinated and we did not decide to give TB preventive chemotherapy. Analyzing our decision in retrospect, it is possible that we might have prevented the development of active TB by introducing preventive chemotherapy. However, preventive chemotherapy has no effect on the overall mortality [22]. The development of drug resistance to M. tuberculosis is also a concern when preventive therapy is given.

The majority of patients with "unmasking" IRIS had a pulmonary form of TB [23]. Only a few cases of TB spondylodiscitis without pulmonary findings have been reported $[15,24]$. The lumbar and lower thoracic regions are most commonly affected in spinal TB [25]. A high index of clinical suspicion and an accurate history of possible TB exposure are essential for the diagnosis of skeletal TB in HIV-infected patients. Spinal TB has no specific diagnostic characteristics or features on radiological imaging and manifests in 2 distinct forms: spondylodiscitis (classic) and spondylitis without disc involvement (atypical) [26]. Involvement of contiguous vertebrae is common [26]. Paraspinal abscesses can develop in more than $50 \%$ of spinal TB cases, and the identification of psoas abscess needs a prompt evaluation of the spine as a primary source of infection [26, 27]. Microscopy and culture of infected material are recommended for the diagnosis of 
spinal TB [25]. Needle aspiration and biopsy, preferably CTguided, are advocated and considered both sensitive and specific [28].

"Paradoxical" reactions generally occur within 1 to 3 months of initiation of treatment [29]. Why "paradoxical" reactions to drug therapy should occur only in some individuals is unclear. It is likely to be due to a complex interplay of the host immune responses, tubercle bacilli virulence, antigen load, the site of infection, and the effects of chemotherapy [30]. The massive delivery of membrane antigens after the initiation of ATT has been advocated as the cause of "paradoxical" responses in immunoincompetent patients [30]. Another possible mechanism is immunologic restoration after antiretroviral therapy and recovery of specific responses to certain antigens [1].

The most frequent risk factors for the development of IRIS found in the majority of studies were starting CART close to the treatment of opportunistic infection, a low CD4 cell count, and a high viral load [31]. In low-income countries, in districts where TB and CART services are not integrated and delivered in different localities, delays in starting CART were largely attributable to the prolonged time between TB diagnosis in the TB clinic and patients access to the CART in the HIV clinic [32, 33].

The treatment of suppurative lymphadenitis and cold abscesses includes drainage of the purulent material. Spontaneous drainage may result in scar formation; hence, repeated punctures or surgical drainage is preferred. We performed US-guided puncture and evacuation of necrotic neck lymph nodes in our patient. The TB abscess of the chest wall was at one time point incised and drained.

According to our experience and the literature search, we recommend the following general principles of treatment based on existing reports, trials, and expert opinions [14, 3439].

(i) Clinicians should become familiar with the case definitions for TB-IRIS and use specific definitions developed for resource-limited settings. Clinicians should consider alternative diagnoses in patients with suspected TB-IRIS, especially if the severity of the condition warrants administering corticosteroids.

(ii) Patients should continue antituberculous therapy without change unless there is a reason to suspect that the current regimen is inadequate.

(iii) In nearly all cases, patient with TB-IRIS should remain on antiretroviral therapy. Certain circumstances, however, may require temporary interruption of antiretroviral therapy, such as life-threatening central nervous system complications of IRIS.

(iv) We recommend performing incision and drainage (with stains and cultures) for localized abscesses, as it was in the case of the psoas abscess in our patient.

(v) Although criteria for initiating corticosteroids remain poorly defined, we recommend administering prednisone for TB-IRIS cases when patients have persistent fever that affects everyday activities. (vi) The dosing and duration of corticosteroids should be tailored to individual patient circumstances.

(vii) The patient receiving corticosteroids for TB-IRIS should undergo careful monitoring for response to corticosteroid therapy and monitoring for the development of possible concurrent infections or other corticosteroid side effects. Prophylaxis for opportunistic infections) should also be given.

(viii) If the patient's clinical condition does not improve, the clinician should consider an alternative diagnosis and possible termination of corticosteroids, since administering corticosteroids to a patient with a non tuberculous opportunistic infection or disease could result in further clinical deterioration.

\section{Acknowledgment}

The authors indicate no potential conflict of interests. There was no financial support for this report.

\section{References}

[1] M. Narita, D. Ashkin, E. S. Hollender, and A. E. Pitchenik, "Paradoxical worsening of tuberculosis following antiretroviral therapy in patients with AIDS," American Journal of Respiratory and Critical Care Medicine, vol. 158, no. 1, pp. 157-161, 1998.

[2] J. E. Fishman, E. Saraf-Lavi, M. Narita, E. S. Hollender, R. Ramsinghani, and D. Ashkin, "Pulmonary tuberculosis in AIDS patients: transient chest radiographic worsening after initiation of antiretroviral therapy," American Journal of Roentgenology, vol. 174, no. 1, pp. 43-49, 2000.

[3] K. A. Wendel, K. S. Alwood, R. Gachuhi, R. E. Chaisson, W. R. Bishai, and T. R. Sterling, "Paradoxical worsening of tuberculosis in HIV-infected persons," Chest, vol. 120, no. 1, pp. 193-197, 2001.

[4] E. Navas, P. Martín-Dávila, L. Moreno et al., "Paradoxical reactions of tuberculosis in patients with the acquired immunodeficiency syndrome who are treated with highly active antiretroviral therapy," Archives of Internal Medicine, vol. 162, no. 1, pp. 97-99, 2002.

[5] I. Puljiz and J. Begovac, "Tuberculosis in HIV-infected patients in Croatia between 1986 and 2005," Collegium Antropologicum, vol. 30, supplement 2, pp. 53-58, 2006.

[6] L. J. Haddow, P. J. Easterbrook, A. Mosam et al., "Defining immune reconstitution inflammatory syndrome: evaluation of expert opinion versus 2 case definitions in a south african cohort," Clinical Infectious Diseases, vol. 49, no. 9, pp. 1424-1432, 2009.

[7] G. Meintjes, S. D. Lawn, F. Scano et al., “Tuberculosis-associated immune reconstitution inflammatory syndrome: case definitions for use in resource-limited settings," The Lancet Infectious Diseases, vol. 8, no. 8, pp. 516-523, 2008.

[8] M. A. French, P. Price, and S. F. Stone, "Immune restoration disease after antiretroviral therapy," Acquired Immunodeficiency Syndrome, vol. 18, no. 12, pp. 1615-1627, 2004.

[9] S. A. Shelburne, M. Montes, and R. J. Hamill, "Immune reconstitution inflammatory syndrome: more answers, more questions," Journal of Antimicrobial Chemotherapy, vol. 57, no. 2, pp. 167-170, 2006. 
[10] R. Colebunders, L. John, V. Huyst, A. Kambugu, F. Scano, and L. Lynen, "Tuberculosis immune reconstitution inflammatory syndrome in countries with limited resources," International Journal of Tuberculosis and Lung Disease, vol. 10, no. 9, pp. 946953, 2006.

[11] C. B. Choremis, C. Padiatellis, D. Zou Mbou Lakis, and D. Yannakos, "Transitory exacerbation of fever and roentgenographic findings during treatment of tuberculosis in children," American Review of Tuberculosis, vol. 72, no. 4, pp. 527-536, 1955.

[12] R. A. M. Breen, C. J. Smith, H. Bettinson et al., "Paradoxical reactions during tuberculosis treatment in patients with and without HIV co-infection," Thorax, vol. 59, no. 8, pp. 704-707, 2004.

[13] I. A. Campbell and A. J. Dyson, "Lymph node tuberculosis: a comparison of various methods of treatment," Tubercle, vol. 58, no. 4, pp. 171-179, 1977.

[14] L. J. Haddow, M. Y. S. Moosa, and P. J. Easterbrook, "Validation of a published case definition for tuberculosis-associated immune reconstitution inflammatory syndrome," Acquired Immunodeficiency Syndrome, vol. 24, no. 1, pp. 103-108, 2010.

[15] G. Meintjes, M. X. Rangaka, G. Maartens et al., "Novel relationship between tuberculosis immune reconstitution inflammatory syndrome and antitubercular drug resistance," Clinical Infectious Diseases, vol. 48, no. 5, pp. 667-676, 2009.

[16] I. Bozicevic and J. Begovac, "The emerging HIV epidemic among men who have sex with men in southeastern Europe," Expert Review of Anti-Infective Therapy, vol. 8, no. 12, pp. 13511358, 2010.

[17] J. Begovac, A. Zekan, and D. Skoko-Poljak, "Twenty years of human immunodeficiency virus infection in Croatia-an epidemic that is still in an early stage," Collegium Antropologicum, vol. 30, no. 2, pp. 17-23, 2006.

[18] T. Franquet, N. L. Müller, A. Giménez, S. Martínez, M. Madrid, and P. Domingo, "Infectious pulmonary nodules in immunocompromised patients: usefulness of computed tomography in predicting their etiology," Journal of Computer Assisted Tomography, vol. 27, no. 4, pp. 461-468, 2003.

[19] A. Kuzucu, O. Soysal, and H. Günen, "The role of surgery in chest wall tuberculosis," Interactive Cardiovascular and Thoracic Surgery, vol. 3, no. 1, pp. 99-103, 2004.

[20] S. Suchindran, E. S. Brouwer, and A. van Rie, "Is HIV infection a risk factor for multi-drug resistant tuberculosis? A systematic review," PLoS One, vol. 4, no. 5, Article ID e5561, 2009.

[21] S. J. Buckingham, L. J. Haddow, P. J. Shaw, and R. F. Miller, "Immune reconstitution inflammatory syndrome in HIVinfected patients with mycobacterial infections starting highly active anti-retroviral therapy," Clinical Radiology, vol. 59, no. 6, pp. 505-513, 2004.

[22] S. Woldehanna and J. Volmink, "Treatment of latent tuberculosis infection in HIV infected persons," Cochrane Database of Systematic Reviews, no. 1, Article ID CD000171, 2004.

[23] M. Tahir, S. Sinha, S. K. Sharma, and R. T. Mitsuyasu, "Immune reconstitution inflammatory syndrome manifesting as disseminated tuberculosis, deep venous thrombosis, encephalopathy and myelopathy," The Indian Journal of Chest Diseases \& Allied Sciences, vol. 50, no. 4, pp. 363-364, 2008.

[24] Y. C. Manabe, R. Breen, T. Perti, E. Girardi, and T. R. Sterling, "Unmasked tuberculosis and tuberculosis immune reconstitution inflammatory disease: a disease spectrum after initiation of antiretroviral therapy," Journal of Infectious Diseases, vol. 199, no. 3, pp. 437-444, 2009.
[25] R. Maron, D. Levine, T. E. Dobbs, and W. M. Geisler, “Two cases of pott disease associated with bilateral psoas abscesses: case report," Spine, vol. 31, no. 16, pp. E561-E564, 2006.

[26] E. Pertuiset, J. Beaudreuil, F. Lioté et al., "Spinal tuberculosis in adults: a study of 103 cases in a developed country, 1980-1994," Medicine, vol. 78, no. 5, pp. 309-320, 1999.

[27] G. Rajeswaran, J. L. Becker, C. Michailidis, A. L. Pozniak, and S. P. G. Padley, "The radiology of IRIS (immune reconstitution inflammatory syndrome) in patients with mycobacterial tuberculosis and HIV co-infection: appearances in 11 patients," Clinical Radiology, vol. 61, no. 10, pp. 833-843, 2006.

[28] A. Mondal, "Cytological diagnosis of vertebral tuberculosis with fine-needle aspiration biopsy," Journal of Bone and Joint Surgery American volume, vol. 76, no. 2, pp. 181-184, 1994.

[29] Y. K. Yoon, J. Y. Kim, J. W. Sohn et al., "Paradoxical response during antituberculous therapy in a patient discontinuing infliximab: a case report," Journal of Medical Case Reports, vol. 3, article 6673, 2009.

[30] H. Bukharie, "Paradoxical response to anti-tuberculous drugs: resolution with corticosteroid therapy," Scandinavian Journal of Infectious Diseases, vol. 32, no. 1, pp. 96-97, 2000.

[31] S. Karmakar, S. K. Sharma, R. Vashishtha et al., "Clinical characteristics of tuberculosis-associated immune reconstitution inflammatory syndrome in North Indian population of HIV/AIDS patients receiving HAART,' Clinical and Developmental Immunology, vol. 2011, Article ID 239021, 9 pages, 2011.

[32] S. D. Lawn, L. Campbell, R. Kaplan et al., "Delays in starting antiretroviral therapy in patients with HIV-associated tuberculosis accessing non-integrated clinical services in a South African township," BMC Infectious Diseases, vol. 11, article 258, 2011.

[33] H. van der Plas, G. Meintjes, C. Schutz et al., "Complications of antiretroviral therapy initiation in hospitalised patients with HIV-associated tuberculosis," PloS One, vol. 8, no. 2, Article ID e54145, 2013.

[34] K. Cohen and G. Meintjes, "Management of individuals requiring antiretroviral therapy and TB treatment," Current Opinion in HIV and AIDS, vol. 5, no. 1, pp. 61-69, 2010.

[35] S. S. Abdool Karim, K. Naidoo, A. Grobler et al., "Integration of antiretroviral therapy with tuberculosis treatment," The New England Journal of Medicine, vol. 365, no. 16, pp. 1492-1501, 2011.

[36] F. X. Blanc, T. Sok, D. Laureillard et al., "Earlier versus later start of antiretroviral therapy in HIV-infected adults with tuberculosis," The New England Journal of Medicine, vol. 365, no. 16, pp. 1471-1481, 2011.

[37] D. V. Havlir, M. A. Kendall, P. Ive et al., "Timing of antiretroviral therapy for HIV-1 infection and tuberculosis," The New England Journal of Medicine, vol. 365, no. 16, pp. 482-491, 2011.

[38] G. Meintjes, R. J. Wilkinson, C. Morroni et al., "Randomized placebo-controlled trial of prednisone for paradoxical tuberculosis-associated immune reconstitution inflammatory syndrome," Acquired Immunodeficiency Syndrome, vol. 24, no. 15, pp. 2381-2390, 2010.

[39] G. Manoharan and C. Behrens, "Diagnosis and Management of Tuberculosis Immune Reconstitution Inflammatory Syndrome (TB-IRIS)," http://depts.washington.edu/ghivaids/reslimited/ case3/index.shtml. 


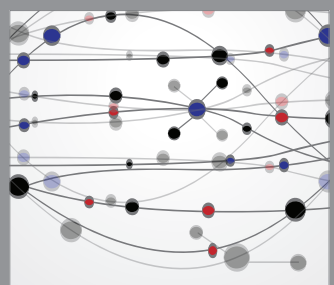

The Scientific World Journal
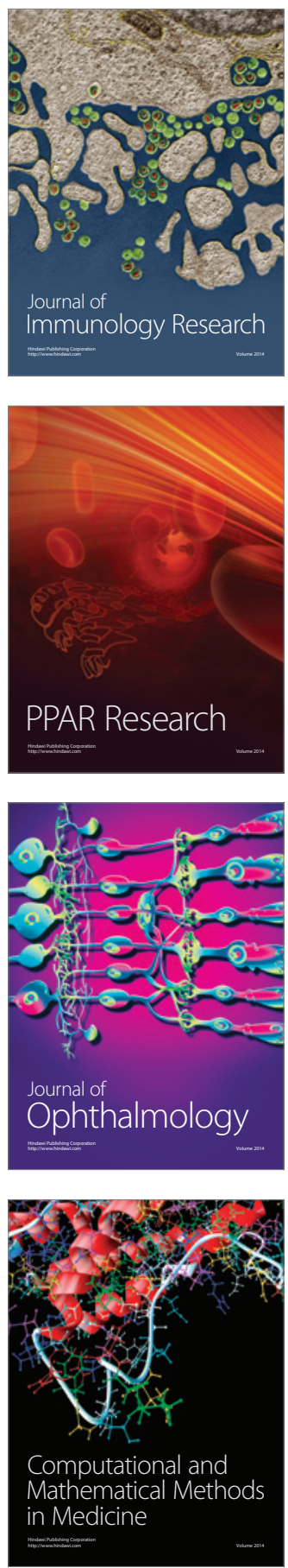

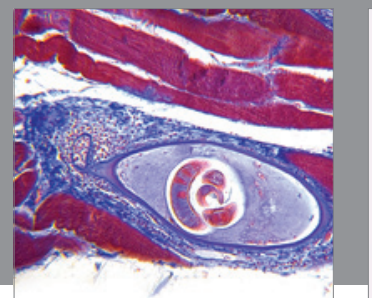

Gastroenterology

Research and Practice
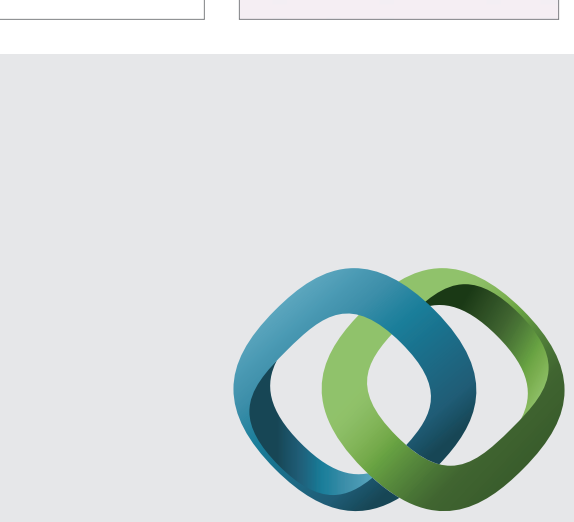

\section{Hindawi}

Submit your manuscripts at

http://www.hindawi.com
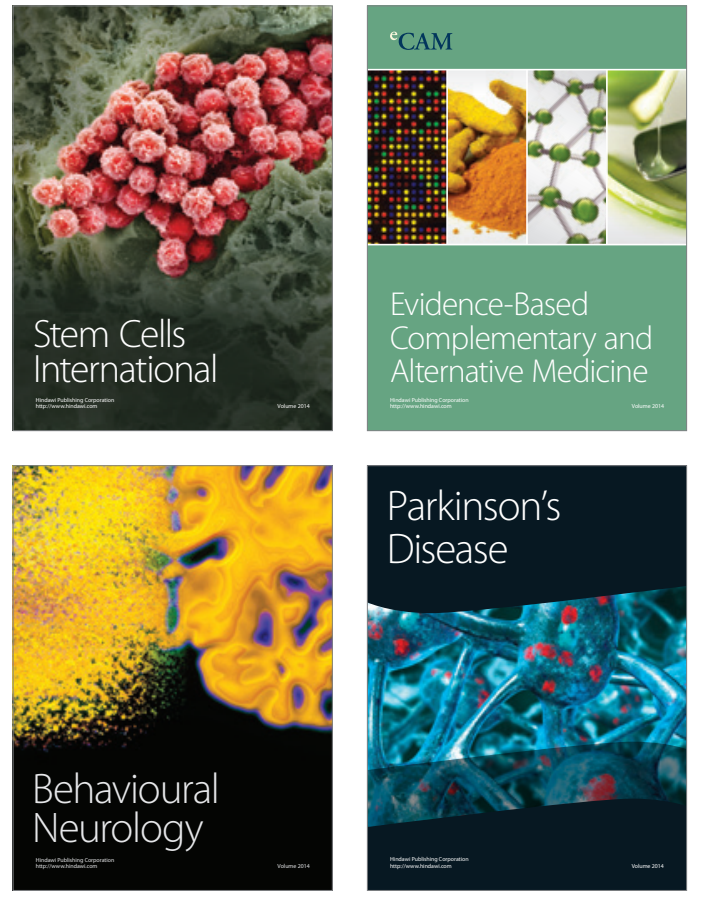
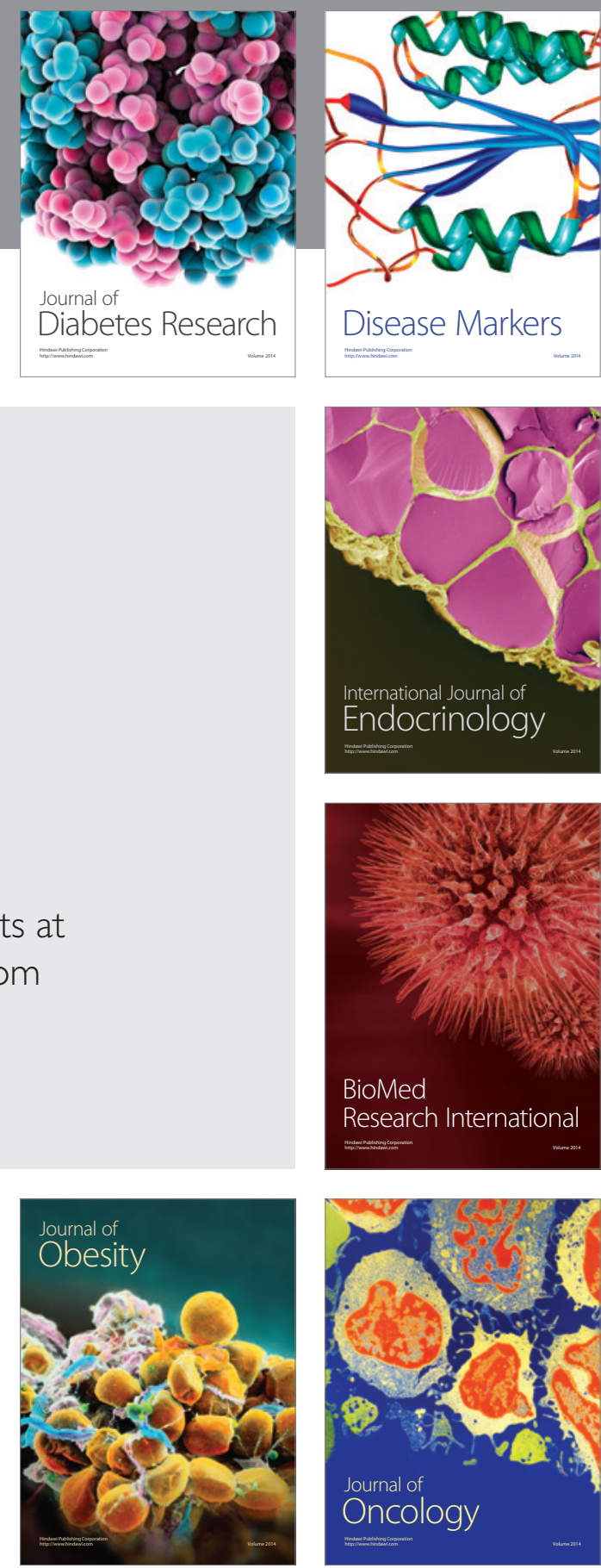

Disease Markers
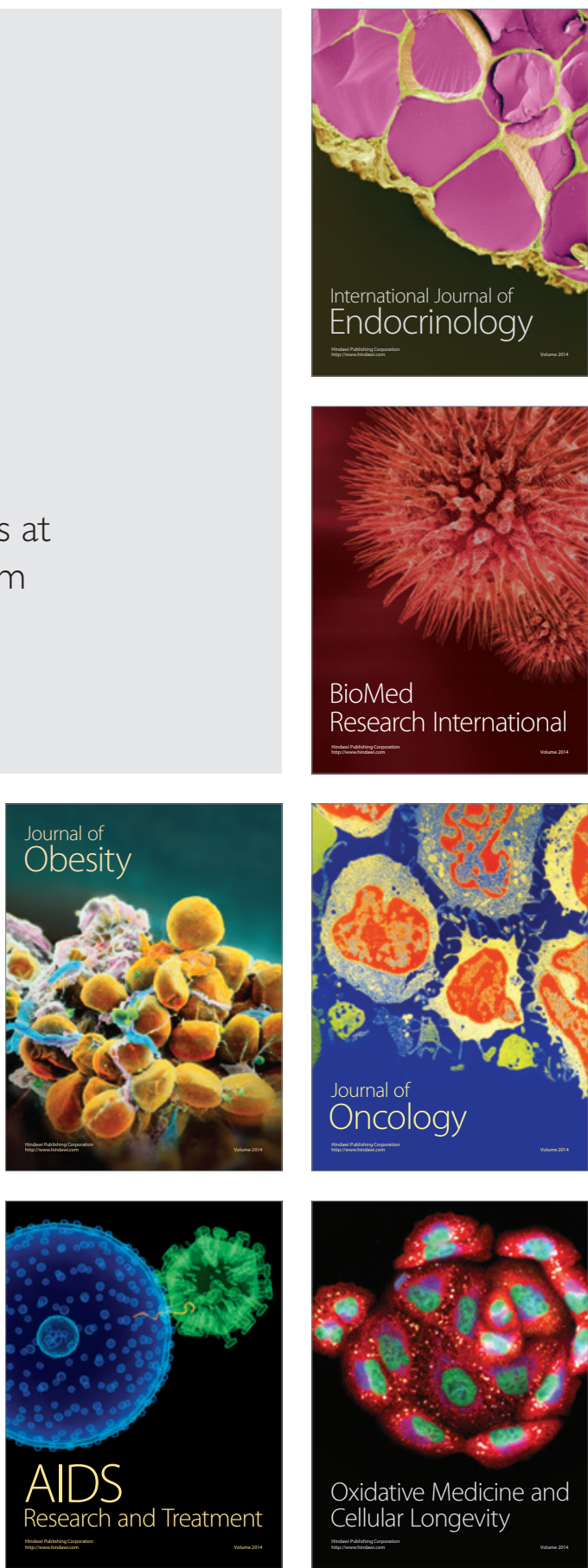